\title{
Descending Problem in Green's Function Approach to Quantum Field Theory
}

\author{
Tetz Yoshimura \\ Department of Mathematics, King's College, London, England
}

Received September 10, 1974

\begin{abstract}
The question how to determine lower many-point functions in terms of higher ones, which we call the descending problem, is discussed for the $\left(\phi^{4}\right)_{1+3}$ model of quantum field theory. Equations to be considered are non-linear non-compact operator equations in complex Banach spaces.

Several sufficient sets of conditions for convergence of successive approximation schemes are presented for small values of the renormalised coupling constant. Local uniqueness of solution is proved under certain conditions.
\end{abstract}

\section{Introduction}

Usually, quantum field theory is concerned with expression of higher many point functions in terms of lower ones. But as will be discussed in Chapter VI, this problem does not seem to have unique solutions even for polynomially nonlinear interactions unless a perturbative approach makes sense. Now let us ask the converse question. Suppose $G_{N}(N$ fixed) were known or substituted by a model function satisfying the causality condition etc. Is it then possible to determine $G_{n}(n<N)$ ? This is a relevant question, because 1) for example, for Yukawa type interaction the lowest observable processes correspond to four point functions; and 2) if one begins with $G^{0}$ (bare propagator) and point vertex, one ends up with divergences and ghosts except in superrenormalisable models. (Here $G_{2}$ stands for the two point function in the Heisenberg representation and $G_{n}(n \geqq 4)$ stand for amputated connected $n$-point functions.)

In this note we pursue the Green's function approach to quantum field theory. By Green's function approach we mean that once the equations for Green's functions (many-point functions) have been derived, one can forget field operators and deal exclusively with Green's functions.

Let us take the $\left(\phi^{4}\right)_{1+3}$ model and suppose that $G_{4}$ is given. Then our problem is concerned with the existence and uniqueness of the following equation:

$$
\begin{aligned}
& G_{2}(p)-\left(p^{2}-m_{r}^{2}-i \varepsilon\right)^{-1} g_{r} \int_{m_{r}^{2}}^{p^{2}} d\left(p^{\prime 2}\right) \int_{m_{r}^{2}}^{p^{\prime 2}} d\left(p^{\prime \prime 2}\right) \frac{d^{2}}{d\left(p^{\prime \prime 2}\right)^{2}} \\
& \cdot \int d^{4} q_{1} d^{4} q_{2} G_{2}\left(q_{1}\right) G_{2}\left(q_{2}\right) G_{2}\left(p^{\prime \prime}-q_{1}-q_{2}\right) G_{4}\left(q_{1}, q_{2}, p^{\prime \prime}-q_{1}-q_{2},-p^{\prime \prime}\right) \\
& \cdot G_{2}(p)=\left(p^{2}-m_{r}^{2}-i \varepsilon\right)^{-1} \equiv G^{0}(p) .
\end{aligned}
$$

This equation incorporates a technique of renormalisation introduced by Taylor [1]. Let us define $\sigma$ as follows

$$
\sigma\left(p^{2}\right)=G_{2}(p)-G^{0}(p) .
$$




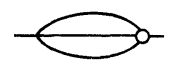

Fig. 1. Selfenergy part $\llbracket G_{2}^{* 3} * O_{4} \rrbracket$

Then the equation to be considered is written in the following form:

where

$$
\Xi[\sigma] \equiv(\Phi-I)[\sigma]=0
$$

$\Phi[\sigma]\left(p^{2}\right)=g_{r} \int_{m_{r}^{2}}^{p^{2}} d\left(p^{\prime 2}\right) \int_{m_{r}^{2}}^{p^{\prime 2}} d\left(p^{\prime \prime 2}\right) \frac{d^{2}}{d\left(p^{\prime \prime 2}\right)^{2}}\left[\left(G^{0}+\sigma\right)^{* 3} * G_{4}\right]\left(p^{\prime \prime 2}\right)$

$\cdot G^{0}(p)\left\{p^{2}-m_{r}^{2}-i \varepsilon+g_{r} \int_{m_{r}^{2}}^{p^{2}} d\left(q^{\prime 2}\right) \int_{m_{r}^{2}}^{q^{\prime 2}} d\left(q^{\prime \prime 2}\right) \frac{d^{2}}{d\left(q^{\prime \prime 2}\right)^{2}}\left[\left(G^{0}+\sigma\right)^{* 3} * G_{4}\right]\left(q^{\prime \prime 2}\right)\right\}^{-1}$.

Here asterisk * is a short hand for convolution (see Fig. 1). From now on we write $\llbracket \ldots \rrbracket\left(p^{2}\right)$ instead of $\int_{m_{r}^{2}}^{p^{2}} d\left(p^{\prime 2}\right) \int_{m_{r}^{2}}^{p^{\prime 2}} d\left(p^{\prime \prime 2}\right) \frac{d^{2}}{d\left(p^{\prime \prime 2}\right)^{2}}[\ldots]\left(p^{\prime \prime 2}\right)$. It is convenient to so normalise $G_{4}$ that $G_{4}(0,0,0,0)=1$. Then our $g_{r}$ corresponds to $g^{2}$ in the conventional normalisation.

Some comments are in order. An advantage of (1.2) over (1.1) is in the fact that $\Phi$ does not have a pole at $p^{2}=m_{r}^{2}$ and is expected to be small for small $g$ and small $\|\sigma\|$ if $G_{4}$ is suitably chosen. If one renormalises $\Phi$ with subtraction constants $Z$ and $\delta m^{2}, \Phi[\sigma]$ has two poles that cancel each other only when $\sigma$ is the solution Eq. (1.2). Neither $G_{2}$ itself nor $\Sigma$ (proper selfenergy part) nor $\left(G^{0}\right)^{-1} G_{2}$ is suitable as object to be found as fixed point of a mapping in a function space because $G_{2}$ and $\sum$ are not bounded while $G_{2}$ and $\left(G^{0}\right)^{-1} G_{2}$ are not small when $g$ is small. The advantage of dealing with $\sigma$ is in the fact that $\sigma\left(p^{2}\right)$ should not have a pole and decreases as $\left|p^{2}\right|^{-1}$ when $\left|p^{2}\right| \rightarrow \infty$. If one evaluates $\Phi[\sigma]$ for a $\sigma$ with $\left|\sigma\left(p^{2}\right)\right|$ $>c \cdot\left|p^{2}\right|^{-1}$, one gets $\Phi[\sigma]\left(p^{2}\right) \sim\left|p^{2}\right|^{-1}$ when $\left|p^{2}\right| \rightarrow \infty$. Therefore the Eqs. (1.2) guarantee the desired asymptotic behavior of $\sigma$ as long as the $G_{4}$ is so chosen that the integral $\llbracket\left(G^{0}=\sigma\right)^{* 3} * G_{4} \rrbracket$ is meaningful, and consequently a function with the asymptotic behavior $\sim\left|p^{2}\right|^{\varepsilon}(\varepsilon>-1)$ cannot be a solution of the Eq. (1.2). (In order to make the integral $\llbracket\left(G^{0}+\sigma\right)^{* 3} * G_{4} \rrbracket$ meaningful for $\sigma$ with $\sigma\left(p^{2}\right) \sim\left|p^{2}\right|^{\varepsilon}$ the $\alpha$ in the condition (1.3) below must be $>1+\varepsilon$.)

Because our Eq. (1.2) is "inhomogeneous", it does not have a trivial solution $\sigma=0$. [For the Eq. (1.2a) to have a trivial solution, $G_{4}$ has to be so chosen that $\left[\left(G^{0}\right)^{* 3} * G_{4}\right]\left(p^{2}\right)=c_{0}+c_{1} p^{2} . c_{0}$ and $c_{1}$ being arbitrary constants. $]$

If one takes $G_{4}$ with asymptotic behaviour

$$
G_{4}\left(p_{1}, p_{2}, p_{3}, p_{4}\right)=O(1) \quad \text { when } \operatorname{Max}\left(\left|p_{i}{ }^{2}\right|\right) \rightarrow \infty
$$

as has been the case in the Landau approximation [Landau, Abrikosov and Khalatnikov (2)], then $\llbracket\left(G^{0}+\sigma\right)^{* 3} * G_{4} \rrbracket\left(p^{2}\right)$ behaves asymptotically as $O\left(\left|p^{2}\right| \log \left(\left|p^{2}\right| / m_{r}^{2}\right)\right)$ and a ghost ensues. So, let us take a more reasonable model for $G_{4}$ with the following asymptotic behaviour:

$$
\begin{aligned}
&\left|G_{4}\left(p_{1}, p_{2}, p_{3}, p_{4}\right)\right| \leqq c \frac{\left|p_{1}{ }^{2}\right|^{\alpha}\left|p_{2}{ }^{2}\right|^{\alpha}\left|p_{3}{ }^{2}\right|^{\alpha}\left|p_{4}{ }^{2}\right|^{\alpha}}{\left(\left|p_{1}{ }^{2}\right|+\left|p_{2}{ }^{2}\right|+\left|p_{3}{ }^{2}\right|+\left|p_{4}{ }^{2}\right|\right)^{4 \alpha+\beta}} \\
&(c>0, \alpha>0, \beta \geqq 0) .
\end{aligned}
$$


Then $\llbracket\left(G^{0}+\sigma\right)^{* 3} * G_{4} \rrbracket\left(p^{2}\right)$ behaves asymptotically as $O\left(\left|p^{2}\right|\right)$ (not $O\left(\left|p^{2}\right|^{1-\beta}\right)$ because of renormalization) without a logarithmic factor so that $\Phi[\sigma](1.2 \mathrm{~b})$ does not have a ghost pole for small $g$ and small $\|\sigma\|$ (the norm will be specified below). Of course, (1.3) is not a necessary condition for the absence of a ghost. Alternatively, one can take, e.g.

$$
\begin{gathered}
\left|G_{4}\left(p_{1}, p_{2}, p_{3}, p_{4}\right)\right| \leqq c^{\prime}\left(\sum_{i=1}^{4} \log \left(\left|p_{i}^{2}\right| / m_{r}^{2}\right)\right)^{-1}\left(\log \sum_{j=1}^{4} \log \left(\left|p_{j}^{2}\right| / m_{r}^{2}\right)\right)^{-1} \\
\ldots\left(\operatorname { l o g } \left(\operatorname { l o g } \left(\ldots\left(\sum_{k=1}^{4} \log \left(\left|p_{k}^{2}\right| / m_{r}^{2}\right) \ldots\right)^{-2} .\right.\right.\right.
\end{gathered}
$$

For the mapping under consideration, most of known methods are not applicable.

Let us discuss some of those methods briefly.

The mapping $\Phi$ is not a compact (completely continuous) mapping of Urysohn type in any metrizable function space because the external variable appears in the operand [Taylor (1)]. No topology has been found so far, in which the mapping $\Phi$ is compact. The non-compactness of the mapping can be more easily seen in the Eq. (1.1): Because $G_{2}$ appears as a factor outside of the integration, the image of a bounded domain of infinite dimension can be neither of finite dimension nor be confined in a Hilbert cube. Therefore, the theory of Fredholm mappings (Elworthy and Tromba (3)) is not applicable.

The mapping is not a contraction mapping, either. Though, for small $g$,

and

$$
\|\Phi[0]-\Phi[\Phi[0]]\|=O\left(g^{2}\right)<\|0-\Phi[0]\|=O(g)
$$

$$
\left\|\Phi^{n}[0]-\Phi^{n+1}[0]\right\|=O\left(g^{n+1}\right),
$$

one cannot verify the following inequality:

$$
\begin{gathered}
\left\|\Phi^{n}[0]-\Phi\left[\alpha \Phi^{n-1}[0]\right]\right\| \leqq(1-\alpha)\left\|\Phi^{n-1}[0]-\Phi^{n-2}[0]\right\| \\
(0<\alpha<1)
\end{gathered}
$$

where

$$
\Phi^{n}[\cdot]=\Phi\left[\Phi^{n-1}[\cdot]\right], \quad \Phi^{1}=\Phi
$$

and $\Phi[0]$ stands for $\Phi$ evaluated on the zero function.

Similarly, it seems impracticable to find a domain on which $\Phi$ is strictly contracting, even if such a domain exists. Therefore one cannot apply the fixed point theorem for holomorphic mappings (Earle and Hamilton [4]).

The so-called $A$-proper approximation scheme (Petryshyn [5], Şchiop [6]) is not applicable, either. The main obstacles are the infinite interval of integration, principal value integrals, expected branch points of solution at thresholds, and the convolution. For example, though the non-linear mapping

$$
K[u](x)=u(x) \int_{0}^{1} d s K(x, s) u^{2}(s)
$$

with a kernel of the form

$$
K(x, s)=\int_{0}^{1} d t L(x, t) L(t, s)
$$


with a continuous symmetric positive-definite $L$, is not completely continuous, one can apply Ritz' method to the integral equation

$$
[I-K] u(x)=f(x), \quad f \in L_{2}(0,1)
$$

which resembles our Eq. (1.1) (for the $\phi^{3}$ model) (S,chiop [6]).

\section{Newton-Kantorovich Scheme}

Now, we come to the Newton-Kantorovich scheme.

Take the general form of operator equation

$$
P(x)=0,
$$

where $P$ is a non-linear operator defined in the sphere $S\left(x_{0}, r\right)=\left\{x \mid\left\|x-x_{0}\right\| \leqq r\right\}$ in a Banach space $\mathbb{X}$ with a range in a Banach space $\mathbb{Y}$. The operator $P$ is assumed to be "smooth" so that it has Fréchet derivatives at least up to the second order in the domain $S\left(x_{0}, r\right)$. If $\left[P^{\prime}\left(x_{0}\right)\right]^{-1}$ can be defined as an linear operator from $P\left[S\left(x_{0}, r\right)\right]$ into $\mathbb{X}$, then one can write the first approximation to the solution of the Eq. (2.1) as follows:

Similarly

$$
x_{1}=x_{0}-\left[P^{\prime}\left(x_{0}\right)\right]^{-1} P\left(x_{0}\right) .
$$

$$
x_{n}=x_{n-1}-\left[P^{\prime}\left(x_{n-1}\right)\right]^{-1} P\left(x_{n-1}\right) .
$$

Then a sufficient set of conditions for convergence of the approximation scheme (2.3) is given as follows:

Theorem 1. Let there be such an element $x_{0} \in S$ for a given mapping $P$ that the following conditions are satisfied:

1) For the element $x_{0}$, the mapping $P^{\prime}\left(x_{0}\right)$ has an inverse $F_{0} \equiv\left[P^{\prime}\left(x_{0}\right)\right]^{-1}$ which is bounded in norm:

$$
\left\|F_{0}\right\| \leqq B_{0}
$$

2) $F_{0} P\left(x_{0}\right)$ is bounded:

$$
\left\|F_{0} P\left(x_{0}\right)\right\| \leqq \eta_{0}
$$

3) The second Fréchet derivative $P^{\prime \prime}(x)$ is uniformly bounded in norm:

$$
\left\|P^{\prime \prime}(x)\right\| \leqq \dot{K} \quad \forall x \in S,
$$

$S$ being defined below;

4) Constants $B_{0}, \eta_{0}$ and $K$ satisfy the condition

$$
B_{0} \eta_{0} K \equiv h_{0}<1 / 2 \text {. }
$$

Then the equation $P(x)=0$ has a solution $x^{*}$ in the sphere defined by

$$
\begin{aligned}
& S=\left\{x \mid\left\|x-x_{0}\right\| \leqq r\right\} \\
& r \equiv N\left(h_{0}\right) \eta_{0} \equiv\left(1-\left(1-2 h_{0}\right)^{1 / 2}\right) h_{0}^{-1} \eta_{0} .
\end{aligned}
$$

And the speed of convergence is given by

$$
\left\|x^{*}-x_{n}\right\| \leqq 2^{1-n}\left(2 h_{0}\right)^{2^{n}-1} \eta_{0} .
$$


This and the Theorem $2 \sim 5$ below are adapted from Jankó's book [7] (see also Vainberg [8]).

Now let us apply this theorem to our descending problem. Let us take Banach space $\mathbb{B}$ of continuous complex-valued functions $\sigma$ defined on $\mathbb{R}$ such that $\left|\sigma\left(p^{2}\right)\right|$ and $\left|p^{2} \sigma\left(p^{2}\right)\right|$ are bounded, and define the norm $\|\cdot\|$ by

$$
\|\sigma\|=\alpha_{1} \sup _{x \in \mathbb{R}}|\sigma(x)|+\alpha_{2} \sup _{y \in \mathbb{R}}|y \sigma(y)| \quad\left(\alpha_{1}, \alpha_{2}>0\right) .
$$

There is no point in taking a more sophisticated norm, because there seems to be no norm with respect to which our mapping is compact.

The first and second Fréchet derivatives of $\Xi$ are

$$
\begin{gathered}
\Xi^{\prime}\left[\xi ; \xi^{\prime}\right] \equiv \Phi^{\prime}\left[\xi ; \xi^{\prime}\right]-I \xi^{\prime} \\
=\frac{3 g \llbracket\left(G^{0}+\xi\right)^{* 2} * \xi^{\prime} * G_{4} \rrbracket}{\left\{\left(G^{0}\right)^{-1}-g \llbracket\left(G^{0}+\xi\right)^{* 3} * G_{4} \rrbracket\right\}^{2}}-I \xi^{\prime} \\
\Xi^{\prime \prime}\left[\xi ; \xi^{\prime}, \xi^{\prime \prime}\right] \equiv \Phi^{\prime \prime}\left[\xi ; \xi^{\prime}, \xi^{\prime \prime}\right]=\frac{6 g \llbracket\left(G^{0}+\xi\right) * \xi^{\prime} * \xi^{\prime \prime} * G_{4} \rrbracket}{\left\{\left(G^{0}\right)^{-1}-g \llbracket\left(G^{0}+\xi\right)^{*} 3 * G_{4} \rrbracket\right\}^{2}} \\
-\frac{18 g^{2} \llbracket\left(G^{0}+\xi\right)^{* 2} * \xi^{\prime} * G_{4} \rrbracket \llbracket\left(G^{0}+\xi\right)^{* 2} * \xi^{\prime \prime} * G_{4} \rrbracket}{\left\{\left(G^{0}\right)^{-1}-g \llbracket\left(G^{0}+\xi\right)^{* 3} * G_{4} \rrbracket\right\}^{3}} .
\end{gathered}
$$

(The second Fréchet derivative is a bilinear operator.) Fréchet derivatives $\Xi^{\prime}\left[\xi ; \xi^{\prime}\right]\left(p^{2}\right)$ and $\Xi^{\prime \prime}\left[\xi ; \xi^{\prime}, \xi^{\prime \prime}\right]\left(p^{2}\right)$ have no poles at $p^{2}=m_{r}^{2}$, because the zero of the denominator is compensated by the zero of the numerators, so that $\left\|\Xi^{\prime}[\xi ; \cdot]\right\|$ and $\left\|\Xi^{\prime \prime}[\xi ; \cdot, \cdot]\right\|$ are bounded for $\xi$ with sufficiently small norm.

To find the first approximation $\sigma_{1}$ from the zeroth approximation $\sigma_{0}=0$ one has to solve a linear integral equation, i.e. to invert the Fréchet derivative. Graphical representation of the first approximation is given in Fig. 2.

By power counting for small $g$ one finds a rough estimation

$$
\left\|\Xi^{\prime}[0 ; \cdot]\right\| \leqq B_{0}=O\left(g^{0}\right)
$$

because $\left\|\Phi^{\prime}[0 ; \cdot]\right\|=O(g),\|I\|=1$ while integrations contribute finite factors. On the other hand $\|\Phi[0]\|=O(g),\|I 0\|=0$ so that

Hence

$$
\begin{aligned}
\left\|F_{0} \Xi[0]\right\| & \leqq \eta_{0}=O(g) \\
\left\|\Xi^{\prime \prime}[\xi ; \cdot, \cdot]\right\| & \leqq K=O(g) \quad \forall \xi \in S(0, r) .
\end{aligned}
$$

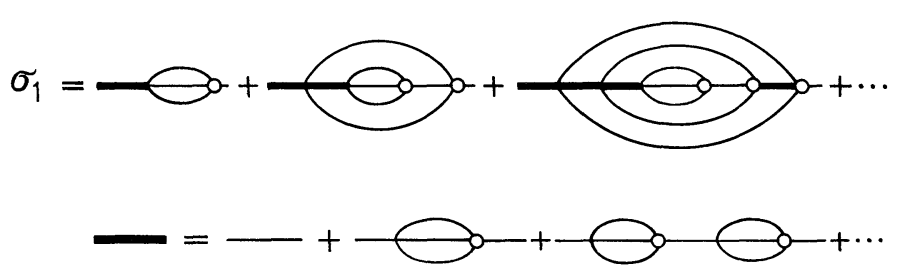

Fig. 2. The first approximation $\sigma_{1}$. Here thin line stands for $G^{0}$ 
i.e. there is a finite interval $\mathscr{J}=\left\{g \mid g_{1}>g>0\right\}$ such that for a value of $g$ in $\mathscr{J}$, $h_{0}<1 / 2$, and consequently the scheme converges. (We need not deal with quasinorms of non-linear operators.) From Fig. 2 one can easily see that $\sigma_{1}$ has already all the expected branch points (thresholds) though their numerical coefficients are modified by higher approximations.

A branch points of the type $\left(p-p_{n}\right)^{\alpha_{n}}\left(\alpha_{n}>0\right)$ does not affect integrability even if it coincides with a simple pole.

As for uniqueness of solution, we have the following theorem:

Theorem 2. Suppose that the following conditions are satisfied: and

1) There exist an element $x_{0} \in \mathbb{X}$ such that $P^{\prime}\left(x_{0}\right)$ has inverse $F_{0}=\left[p^{\prime}\left(x_{0}\right)\right]^{-1}$

$$
\begin{array}{r}
\left\|F_{0}\right\| \leqq B ; \\
\left\|F_{0} P\left(x_{0}\right)\right\| \leqq \eta_{0}
\end{array}
$$

3) Lipschitz condition

$\left\|P^{\prime}\left(x_{1}\right)-P^{\prime}\left(x_{2}\right)\right\| \leqq K\left\|x_{1}-x_{2}\right\| \forall x_{1}, x_{2} \in S \equiv\left\{x \mid\left\|x-x_{0}\right\|<2 \eta_{0}\right\} \subset \mathbb{X} ;$

4)

$$
h_{0}=B_{0} K \eta_{0} \leqq 1 / 2 \text {. }
$$

Then the equation $P(x)=0$ has only one solution in the sphere $S$.

For the descending problem, one gets the following estimation:

$$
B_{0}=O\left(g^{0}\right), \eta_{0}=O(g), K=O(g)
$$

so that one finds $h_{0}=O\left(g^{2}\right)<\frac{1}{2}$ for sufficiently small values of $g$, and consequently the solution is unique in $S$. This theorem, however, does not guarantee global uniqueness of solutions. For global uniqueness one has to use Altman's method [9] of contraction with non-linear majorant. We shall come back to this question in Chapter IV.

\section{Approximation Schemes of Order 3}

There are approximation schemes involving the second Fréchet derivatives. Let us consider the scheme

$$
x_{n+1}=x_{n}-F_{n} P\left(x_{n}\right)-\frac{1}{2} F_{n} P^{\prime \prime}\left(x_{n}\right)\left[F_{n} P\left(x_{n}\right)\right]^{* 2}
$$

which is called the method of tangential parabolas. We have the following theorem:

Theorem 3. Let the following conditions be satisfied:

1) $F(x)=\left[P^{\prime}(x)\right]^{-1}$ exists for any $x \in S\left(x_{0}, r\right)$ and is bounded

$$
\|F(x)\| \leqq B
$$

3)

$$
\left\|F_{0} P\left(x_{0}\right)\right\| \leqq \eta_{0}
$$

4)

$$
\begin{gathered}
\left\|P^{\prime \prime}(x)\right\| \leqq M, \quad\left\|P^{\prime \prime \prime}(x)\right\| \leqq K \quad \forall x \in S\left(x_{0}, r\right) ; \\
h_{0} k_{0}=B M \eta_{0} k_{0}<1,
\end{gathered}
$$

where

$$
k_{0}=\left\{\frac{1}{2}\left(1+h_{0} / 4\right)+\frac{1}{6} K B^{-1} M^{-2}\left(1+h_{0} / 2\right)^{3}\right\}^{1 / 2} .
$$


Then the scheme (3.1) converges to the solution $x^{*}$ of the equation $P(x)=0$ and the speed of convergence is

where

$$
\left\|x^{*}-x_{n}\right\| \leqq r\left(h_{0} k_{0}\right)^{3^{n}-1}
$$

$$
r=\left(1+h_{0} / 2\right) \eta_{0} \sum_{n=0}^{\infty}\left(h_{0} k_{0}\right)^{3^{n-1}} .
$$

For the descending problem (1.2), each step $x_{n} \rightarrow x_{n+1}$ amounts to solving two linear integral equations consecutively. It can be easily seen that the third Fréchet derivative

$$
\begin{aligned}
& \Xi^{\prime \prime \prime}\left[\xi ; \xi_{1}, \xi_{2}, \xi_{3}\right] \equiv \Phi^{\prime \prime \prime}\left[\xi ; \xi_{1}, \xi_{2}, \xi_{3}\right] \\
&= \frac{6 g \llbracket \xi_{1} * \xi_{2} * \xi_{3} * G_{4} \rrbracket}{\left\{\left(G_{0}\right)^{-1}-g \llbracket\left(G^{0}+\xi\right)^{* 3} * G_{4} \rrbracket\right\}^{2}} \\
&-\frac{36 g^{2} \llbracket\left(G^{0}+\xi\right)^{* 2} * \xi_{1} * G_{4} \rrbracket \llbracket\left(G^{0}+\xi\right) * \xi_{2} * \xi_{3} * G_{4} \rrbracket}{\left\{\left(G_{0}\right)^{-1}-g \llbracket\left(G^{0}+\xi\right)^{* 3} * G_{4} \rrbracket\right\}^{3}}-\text { perm. } \\
&-\frac{162 g^{3} \llbracket\left(G^{0}+\xi\right)^{* 2} * \xi_{1} * G_{4} \rrbracket \llbracket\left(G^{0}+\xi\right)^{* 2} * \xi_{2} * G_{4} \rrbracket \llbracket\left(G^{0}+\xi\right)^{*} * \xi_{3} * G_{4} \rrbracket}{\left\{\left(G^{0}\right)^{-1}-g \llbracket\left(G^{0}+\xi\right)^{* 3} * G_{4} \rrbracket\right\}^{4}}
\end{aligned}
$$

is also bounded as a trilinear operator for small $g$ and small $\|\xi\|$. For small $g$ and $\sigma_{0}=0$, one gets the following rough estimation:

$$
\left.\begin{array}{lll}
B=O\left(g^{0}\right), & \eta_{0}=O(g), & M=O(g) \\
K=O(g), & h_{0}=O\left(g^{2}\right), & k_{0}=O\left(g^{-1 / 2}\right),
\end{array}\right\}
$$

so that one finds

$$
h_{0} k_{0}=O\left(g^{3 / 2}\right)<1 .
$$

Another scheme called the method of tangential hyperbolas is defined by the following formula (Jankó [7], [10], [11], Collatz [12], Mirakov [13], Jankó and Balazs [14], Balasz and Jankó [15])

where

$$
x_{n+1}=x_{n}-\left[I-F_{n} P^{\prime \prime}\left(x_{n}\right)\left[F_{n} P\left(x_{n}\right)\right]\right]^{-1} F_{n} P\left(x_{n}\right) \text {, }
$$

$$
F_{n}=\left[P^{\prime}\left(x_{n}\right)\right]^{-1} \text {. }
$$

For this scheme we have the following theorem:

Theorem 4. Let the following conditions be satisfied:

1)

$$
\begin{gathered}
\| F(x) \leqq B<\infty \quad \forall x \in S\left(x_{0}, k \eta\right) \\
K \equiv \sum_{n=0}^{\infty}(h f)^{3^{n-1}}>1
\end{gathered}
$$

2)

$$
\left\|x_{1}-x_{0}\right\| \leqq \eta<\infty
$$

3)

$$
\left\|P^{\prime \prime}(x)\right\|<M, \quad\left\|P^{\prime \prime \prime}(x)\right\| \leqq N, \quad \forall x \in S\left(x_{0}, k \eta\right) ;
$$

$$
h f<1 \text {; }
$$


where

$$
\begin{gathered}
h=B \eta M<1, \\
f=(1-h / 2)^{-1 / 2}\left\{(4-2 h)^{-1}+N\left(6 B M^{2}\right)^{-1}\right\}^{1 / 2} .
\end{gathered}
$$

Then there is a solution $x^{*}$ of the equation $P(x)=0$ in the sphere $S\left(x_{0}, K \eta\right)$ and the speed of convergence is

$$
\left\|x^{*}-x_{n}\right\| \leqq K \eta(h f)^{3^{n}-1} .
$$

For our descending problem with a small $g$ one gets the following estimations

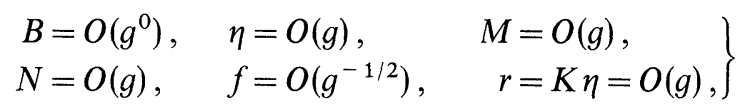

so that for sufficiently small values of $g$ one finds

$$
h f=O\left(g^{3 / 2}\right)<1 \text {. }
$$

We have a useful theorem for even more general schemes of successive approximations of the form

$$
\begin{gathered}
x_{n+1}=x_{n}-\left[I-\left(I-\lambda A_{n}\right)^{-1} \mathrm{~A}_{n}\right] F_{n} P\left(x_{n}\right), \\
A_{n}=\frac{1}{2}\left[F_{n} P^{\prime \prime}\left(x_{n}\right) F_{n} P\left(x_{n}\right)\right] .
\end{gathered}
$$

( $A_{n}$ is a linear operator.) The scheme (3.22) is reduced to the method of tangential hyperbolas, the method of tangential parabolas and Vohandu's method when $\lambda=-1,0,-2$, respectively (Jankó [7]).

Theorem 5. Suppose that the following conditions are satisfied:

1) $F(x) \equiv\left[P^{\prime}(x)\right]^{-1}$ exists for any $x \in S$ and is bounded:

where

$$
\|F\| \leqq B
$$

$$
\begin{gathered}
S=\left\{x \mid x \in \mathbb{X},\left\|x_{0}-x\right\|<\left\{2+(1-|\lambda|) h_{0} \eta_{0}\right\} H\left(2-|\lambda| h_{0}\right)^{-1}\right\} \\
H=\sum_{n=0}^{\infty}\left(h_{0} k_{0}\right)^{3^{n}-1} ; \\
\text { 2) } \quad\left\|F_{0} P\left(x_{0}\right)\right\|<\eta_{0} ; \\
\text { 3) } \quad\left\|P^{\prime \prime}(x)\right\| \leqq M, \quad\left\|P^{\prime \prime}\left(x_{1}\right)-P^{\prime \prime}\left(x_{2}\right)\right\| \leqq K\left\|x_{1}-x_{2}\right\| \forall x, x_{1}, x_{2} \in S \\
\text { 4) } \\
h_{0} k_{0}<1,
\end{gathered}
$$

2)$$
\text { 4) }
$$

where

$$
\begin{aligned}
k_{0}= & \left(2-|\lambda| h_{0}\right)^{-1}\left[\frac{1}{2}(1+|1+\lambda|)\left(2-|\lambda| h_{0}\right)+\frac{1}{2} h_{0}\right. \\
& +2\left\{2+\left(1-|\lambda| h_{0}\right)^{3} K\left\{6 M B\left(2-|\lambda| h_{0}\right)\right\}^{-1}\right]^{1 / 2} .
\end{aligned}
$$

Then the scheme converges to a solution $x^{*}$ of the equation $P(x)=0$, and the speed of convergence is

$$
\left\|x^{*}-x_{n}\right\| \leqq\left\{2+(1-|\lambda|) h_{0}\right\} \eta_{0} H\left(2-|\lambda| h_{0}\right)^{-1}
$$


If this theorem is applied to our descending problem, one gets, for small $g$ following estimate:

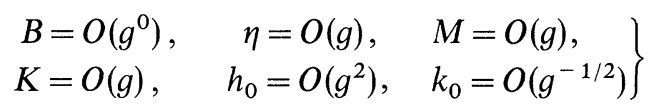

unless $|\lambda|$ is very large, so that one can except

$$
h_{0} k_{0}=O\left(g^{3 / 2}\right)<1,
$$

namely, convergence of the scheme.

\section{Is the Solution Globally Unique?}

So far we have been concerned with the existence and local uniqueness of a solution with small norm for small values of the effective coupling constant. In order to insure the absence of ghost in $\Phi[\sigma]$, it appears that the norm of $\sigma$ need not be small for small values of $g$. Unfortunately, the Theorem 2 does not, however, imply global uniqueness of solutions. $\sigma$ must tend to zero as $g \rightarrow 0$ because $g G_{4}$ ought to tend to zero. For otherwise irreducible higher many point functions would blow up. This, however, does not imply that $\sigma$ and $G_{4}$ must be analytic in $g$ at $g=0$. Rather, to ensure the existence of a solution to the Eq. (1.2), $G_{4}$ should be specified so as to make the integral $\llbracket G_{2}^{* 3} * G_{4} \rrbracket$ behave decently for a given finite value of $g$, not for the whole interval $0 \leqq g \leqq g_{0}<\infty$. On the other hand, for a small value of $g$, the expression

$$
\mathscr{D}\left(g ; \sigma ; p^{2}\right) \equiv p^{2}-m_{r}^{2}-i \varepsilon-g \llbracket\left(G^{0}+\sigma\right)^{*^{3}} * G_{4} \rrbracket
$$

does not have a zero other than at $p^{2}=m_{r}^{2}$ for $\sigma$ 's with relatively large norms and Im $\mathscr{D}$ remains negative definite. It appears that, roughly speaking, the admissible values of $\|\sigma\|$ increase as $\sim g^{-1 / 3}$ when $g$ tends to zero. If one takes a zeroth approximation $\sigma_{0}$ with $\left\|\sigma_{0}\right\|=O\left(g^{-1 / 3}\right)$, then one finds

$$
B=O\left(g^{0}\right), \quad \eta_{0}=O\left(g^{0}\right), \quad K=O\left(g^{1 / 3}\right),
$$

so that one can expect $h_{0}=O\left(g^{1 / 3}\right)<1 / 2$.

To answer the question whether a successive approximation starting from a zeroth approximation with norm $\sim O\left(g^{-1 / 3}\right)$, the most powerful method so far available is Altman's method of contractor with non-linear majorant [9].

Theorem 6. Let $P$ be a closed non-linear operator with domain $\mathbb{D} \subset \mathbb{X}$ and range in $\mathrm{Y}$, and $F(x)$ be a linear operator satisfying the contractor inequality

$$
\|P[x+F(x) y]-P[x]-y\| \leqq a\|y\|^{2}+b\|y\|
$$

for $x \subset \mathbb{D}$ whenever $x+F(x) y \in \mathbb{D}$. If a zeroth approximation is chosen so as to satisfy the conditions

$$
\begin{gathered}
S\left(x_{0}, B t^{*}\right) \subset \mathbb{D}, \quad\left\|P x_{0}\right\| \leqq \eta, \quad t^{*}=\eta(1-q)^{-1} ; \\
\|F(x)\| \leqq B \forall x \in S \subset \mathbb{D} ; \\
q=a y+b<1 .
\end{gathered}
$$


Then the approximation scheme

$$
x_{n+1}=x_{n}-F\left(x_{n}\right) P\left(x_{n}\right)
$$

converges to a solution $x^{*}$ of the equation $P(x)=0$ and the error estimation is

$$
\left\|x^{*}-x_{n}\right\| \leqq B \eta q^{n}(1-q)^{-1} \text {. }
$$

If one applies this theorem to the Eq. (1.2), one finds that the inequality (4.3) is satisfied by $a$ and $b$ chosen subject to the conditions

whenever

$$
\left.\begin{array}{ll}
a \leqq O\left(g^{2 / 3}\right), & b \leqq O\left(g^{1 / 3}\right), \\
F[\sigma]=\Xi^{\prime}[\sigma], & \|F\| \leqq B=O\left(g^{0}\right),
\end{array}\right\}
$$

$$
\sigma+F[\sigma] \eta \in \mathbb{D}[I+\Phi]=\left\{\sigma \mid\|\sigma\| \leqq f g^{-1 / 3}\right\}
$$

where $f$ is a constant that depends on details of $G_{4}$. Take a zeroth approximation $\sigma_{0}$ with norm $\sim O\left(g^{-\gamma}\right)\left(0<\gamma<\frac{1}{3}\right)$ and a sphere

where

$$
\Im \equiv\left\{\sigma \mid\left\|\sigma-\sigma_{0}\right\|<B \eta(1-\zeta)^{-1}\right\} \subset \mathbb{D}[I+\Phi] \text {, }
$$

$$
\begin{aligned}
& \left\|[I+\Phi] \sigma_{0}\right\| \leqq \eta=O\left(g^{-\gamma}\right), \\
& \zeta=a \eta+b=O\left(g^{1-2 \gamma}\right)<1 .
\end{aligned}
$$

Then one gets the following estimate for the first approximation:

$$
\left\|\sigma_{1}\right\|=O\left(g^{1-3 \gamma}\right)<1,
$$

while the error estimate reads

$$
\left\|\sigma_{1}-\sigma^{*}\right\| \leqq B \eta \zeta(1-\zeta)^{-1}=O\left(g^{1-3 \gamma}\right)<1,
$$

where $\sigma^{*}$ is the solution of the Eq. (1.2). For higher approximation one finds

$$
\left\|\sigma_{n}\right\|=O(g) \quad(n \geqq 2) .
$$

Whether the sequence $\sigma_{n}$ converges to the solution obtained from zeroth approximation $\sigma_{0}=0$ depends on whether the new $\sigma_{n}$ eventually enter the sphere $S$ defined in the Theorem 2. On the other hand, there cannot be a solution with norm $\left\|\sigma^{*}\right\|=O\left(g^{0}\right)$. There may or may not be "large" solutions with norm $\sim O\left(g^{-1 / 3}\right)$. But one cannot apply any known method to search for them.

\section{Descending Problem from Higher Many Point Functions}

For descending problems from higher many-point functions, the situation is quite different. Suppose $G_{6}$ were known or substituted by a model function. Then the relevant equations are

$$
\begin{aligned}
& \sigma=\frac{g G^{0} \llbracket\left(G^{0}+\sigma\right)^{* 3} * G_{4} \rrbracket}{\left(G^{0}\right)^{-1}-g \llbracket\left(G^{0}+\sigma\right)^{* 3} * G_{4} \rrbracket}, \\
& \mathrm{G}_{4}=-\phi-1-++\longrightarrow \alpha+\infty
\end{aligned}
$$


with appropriate regularisations (Taylor and Yoshimura [16])

$$
\left[\left(G^{0}+\sigma\right)^{* 2} * G_{4}\right]\left(p_{1}, \ldots, p_{4}\right) \rightarrow \prod_{i=1}^{4} D_{i}^{-1} D_{i}\left[\left(G^{0}+\sigma\right)^{* 2}\right]\left(p_{1}, \ldots, p_{4}\right)
$$

etc., where

$$
D_{i}=p_{i \mu} \frac{\partial}{\partial p_{i \mu}} \quad(\text { no summation over } i)
$$

and the operation $D^{-1}$ is to be carried out in such a way that the resultant expressions are equal to 0 for zero momenta $(0,0,0,0)$. This time, the unknown is the pair $\left\{\sigma, G_{4}\right\}$.

For the Eq. (5.1) to have a ghostfree solution, $G_{4}$ must behave in such a way that the contribution of the constant term 1 in the r.h.s. of the Eq. (5.2) be asymptotically cancelled by other terms. But this is a very strict condition and one cannot proceed from a zeroth approximation $\left\{\sigma_{0}, G_{4}^{(0)}\right\}$ with $\sigma_{0}=0$ and a $G_{4}^{(0)}$ such that asymptotically e.g.

$$
\left|G_{4}^{(0)}\left(p_{1}, \ldots, p_{4}\right)\right| \leqq C \frac{\left|p_{1}{ }^{2}\right|^{\alpha}\left|p_{2}{ }^{2}\right|^{\alpha}\left|p_{3}{ }^{2}\right|^{\alpha}\left|p_{4}{ }^{2}\right|^{\alpha}}{\left(\left|p_{1}{ }^{2}\right|+\left|p_{2}{ }^{2}\right|+\left|p_{3}{ }^{2}\right|+\left|p_{4}{ }^{2}\right|\right)^{4 \alpha+\beta}} \quad(\alpha>0, \beta>0) .
$$

If such a $G_{4}^{(0)}$ is substituted into the r.h.s. of the Eq. (5.2), the resulting expression does not enjoy the same asymptotic behaviour as $G_{4}^{(0)}$, so that one cannot proceed further, though a solution may exist.

On the other hand, if $G_{6}$ corresponding to a given pair $\left\{\sigma, G_{4}\right\}$ were known, one could ask whether $G_{2}$ and $G_{4}$ are unique for the given $G_{6}^{\text {irred }}$.

Now, let us define a Banach space $\mathfrak{B}$ of pairs $\left\{\sigma, G_{4}\right\}$ with the norm

$$
\begin{gathered}
\left\|\left\{\sigma, G_{4}\right\}\right\|_{\mathfrak{B}}=\gamma_{1}\|\sigma\|+\gamma_{2}\left\|G_{4}\right\|_{(4)}, \quad \gamma_{1}, \gamma_{2}>0, \\
\left\|G_{4}\right\|_{(4)}=\delta_{1} \sup _{p_{i}^{2} \in \mathbb{R}}\left|G_{4}\left(p_{1}(i=1, \ldots, 4)\right)\right|+\delta_{2} \sup _{p_{j}^{2} \in \mathbb{R}}\left|G_{4}\left(p_{j}\right)\right|\left(\sum_{k=1}^{4}\left|p_{4}{ }^{2}\right|\right)^{4 \alpha+\beta} \\
+\left.\delta_{3} \sup _{p_{l}^{2} \in \mathbb{R}}\left|G_{4}\left(p_{l}\right) \sum_{j}\right| p_{j}^{2}\right|^{4 \alpha+\beta} \prod_{k=1}^{4}\left(\left|p_{k}^{2}\right|+m_{r}^{2}\right)^{-\alpha} \mid
\end{gathered}
$$

Then the problem is whether the following system of equations has a non-trivial solution $\{\tau, \Delta\} \neq\{0,0\}$ in $\mathfrak{B}$ :

$$
\begin{aligned}
& T\left[G_{4}, \sigma ; \tau, \Delta\right] \\
& \begin{array}{l}
\equiv \frac{g G^{0}\left(\llbracket\left(G^{0}+\sigma\right)^{* 3} * G_{4} \rrbracket-\llbracket\left(G^{0}+\sigma+\tau\right)^{* 3} *\left(G_{4}+\Delta\right) \rrbracket\right)}{\left\{\left(G^{0}\right)^{-1}-g \llbracket\left(G_{0}+\sigma\right)^{* 3} * G_{4} \rrbracket\right\}\left\{\left(G^{0}\right)^{-1}-g \llbracket\left(G^{0}+\sigma+\tau\right)^{* 3} *\left(G_{4}+\Delta\right)\right\}}=\tau . \\
U\left[G_{6}^{\text {irred }}, G_{4}, \sigma ; \tau, \Delta\right] \equiv g^{1 / 2} \llbracket\left(G^{0}+\sigma+\tau\right)^{* 2} *\left(G_{4}+\Delta\right) \rrbracket \\
\quad+g \llbracket\left(G^{0}+\sigma+\tau\right)^{* 4} *\left(G_{4}+\Delta\right)^{* 2} \rrbracket+g \llbracket\left(G^{0}+\sigma+\tau\right)^{* 3} * G_{6}^{\text {irred }} \rrbracket \\
\quad-g^{1 / 2} \llbracket\left(G^{0}+\sigma\right)^{* 2} * G_{4} \rrbracket-g \llbracket\left(G^{0}+\sigma\right)^{* 4} * G_{4}^{* 2} \rrbracket \\
-g \llbracket\left(G^{0}+\sigma\right)^{* 3} * G_{6}^{\text {irred }} \rrbracket=\Delta .
\end{array}
\end{aligned}
$$


Fréchet derivative of the pair of operators $\{T, U\}$ is written in the form of a super matrix:

$$
\begin{aligned}
& \{T, U\}^{\prime}\left[\tau_{1}, \Delta_{1}\right]=\left[\begin{array}{ll}
T,_{1}\left[G_{4}, \sigma ; \tau_{1}, \Delta_{1}\right] & T,_{2}\left[G_{4}, \sigma ; \tau_{1}, \Delta_{1}\right] \\
U,_{1}\left[G_{6}^{\text {irred }}, G_{4}, \sigma ; \tau_{1}, \Delta_{1}\right] & U,_{2}\left[G_{6}^{\text {irred }}, G_{4}, \sigma ; \tau_{1}, \Delta_{1}\right]
\end{array}\right] \\
& T,,_{1}\left[G_{4}, \sigma ; \tau_{1} \Delta_{1}\right]=\frac{g \llbracket\left(G^{0}+\sigma+\tau_{1}\right)^{* 2} * \cdot *\left(G_{4}+\Delta\right) \rrbracket}{\left\{\left(G^{0}\right)^{-1}-g \llbracket\left(G^{0}+\sigma+\tau_{1}\right)^{* 3} *\left(G_{4}+\Delta\right) \rrbracket\right\}^{2}} \\
& T,_{2}\left[G_{4}, \sigma ; \tau_{1}, \Delta_{1}\right]=\frac{g \llbracket\left(G^{0}+\sigma+\tau_{1}\right)^{* 3} * \cdot \rrbracket}{\left\{\left(G^{0}\right)^{-1}-g \llbracket\left(G^{0}+\sigma+\tau_{1}\right)^{* 3} *\left(G_{4}+\Delta\right) \rrbracket\right\}^{2}} \\
& U,{ }_{1}\left[G_{6}^{\text {irred }}, G_{4}, \sigma ; \tau_{1}, \Delta_{1}\right]=2 g^{1 / 2} \llbracket\left(G^{0}+\sigma+\tau_{1}\right) * \cdot *\left(G_{4}+\Delta_{1}\right) \rrbracket \\
& +4 g \llbracket\left(G^{0}+\sigma+\tau_{1}\right)^{* 3} * \cdot *\left(G_{4}+\Delta_{1}\right) * 2 \rrbracket \\
& +3 g \llbracket\left(G^{0}+\sigma+\tau_{1}\right)^{* 2} * \cdot * G_{6}^{\mathrm{irred}} \rrbracket \text {, } \\
& U,_{2}\left[G_{6}^{\text {irred }}, G_{4}, \sigma ; \tau_{1}, \Delta_{1}\right]=g^{1 / 2} \llbracket\left(G^{0}+\sigma+\tau_{1}\right)^{* 2} * \cdot \rrbracket \\
& +2 g \llbracket\left(G^{0}+\sigma+\tau_{1}\right)^{* 4} *\left(G_{4}+\Delta_{1}\right) * \cdot \rrbracket \text {. }
\end{aligned}
$$

These expressions are rather symbolical. As for the orders of convolutions, one has to refer to the Eq. (5.2). Let us define the norm of the super matrix by

$$
\left\|\{T, U\}^{\prime}\right\|=\alpha_{1}\left\|T,_{1}\right\|+\alpha_{2}\left\|T,_{2}\right\|+\beta_{1}\left\|U,_{1}\right\|+\beta_{2}\left\|U,_{2}\right\|
$$

where $\alpha$ 's and $\beta$ 's are positive constants with orders of magnitudes of appropriate powers of $m_{r}$. Then one can apply the Theorems $1 \sim 5$. Let us take a zeroth approximation with norm $\sim O(g)$. Then by the Theorem 3 one gets

$$
\left.\begin{array}{lll}
\left\|\left(\{T, U\}^{\prime}-I\right)^{-1}\right\| \leqq B=O\left(g^{0}\right), & \eta_{0}=O\left(g^{1 / 2}\right), \\
M=O\left(g^{1 / 2}\right), & K=O\left(g^{1 / 2}\right), & h_{0}=O(g), \\
k_{0}=O\left(g^{-1 / 4}\right), & r=O\left(g^{1 / 2}\right) &
\end{array}\right\}
$$

so that for sufficiently small values of $G$ one finds

$$
h_{0} k_{0}=O\left(g^{3 / 4}\right)<1
$$

again. Therefore, if one begins with a sufficiently small zeroth approximation, successive approximation leads to the trivial solution because of the local uniqueness (Theorem 2).

If non-trivial solutions of the Eqs. (5.8), (9) exist at all, they must not have arbitrarily small norms, i.e. "distance" between two sets of $\sigma, G_{4}$ corresponding to a given $G_{6}^{\text {irred }}$ cannot be arbitrarily small.

Again unfortunately, we cannot say anything about existence or non-existence of solutions with large norms.

\section{Ascending Problem}

As a converse of the descending problem, let us consider the ascending problem briefly. If $G_{n}(n \leqq N)$ were known or substituted by model functions, can one determine $G_{N+2}$ by Green's function equations without resorting to perturbative 
expansions? Suppose $G_{2}$ were given, then $G_{4}$ in the $\phi^{4}$ theory must satisfy the following condition:

$$
\left(G_{2}(p)\right)^{-1}-p^{2}+m_{r}^{2}=g \llbracket G_{2}^{* 3} * G_{4} \rrbracket\left(p^{2}\right) .
$$

Then, the question is whether $\Gamma=0$ is the only solution satisfying the conditions of the symmetry, causality etc. of the homogeneous equation

$$
\llbracket G_{2}^{* 3} * \Gamma \rrbracket=0
$$

or equivalently

$$
\left[G_{2}^{* 3} * \Gamma\right]\left(p^{2}\right)=c_{0}+c_{1} p^{2},
$$

$c_{0}$ and $c_{1}$ being arbitrary constants. Of course, $\Gamma$ need not have branch points at thresholds. If the Eq. (6.2) has solutions $\Gamma_{n}$, then any function of the form

$$
G_{4}^{\prime}=G_{4}+\sum_{n} \zeta_{n} \Gamma_{n} \quad\left(\zeta_{n} \in \mathbb{R}\right)
$$

is a solution of the Eq. (6.1). Therefore, if the Eq. (6.2) has at least one non-trivial solution, $\Gamma_{1}$, the Eq. (6.1) has a continuum of solutions. Suppose that a particular solution (four-point function) has been somehow chosen from among infinitely many possibilities. Then the question becomes whether there are irreducible sixpoint functions that satisfy appropriate unitarity, causality and symmetry conditions and the Eq. (5.2). This equation is linear in $G_{6}^{\text {irred }}$ but non-linear in $G_{4}$, so that one cannot separate the effects of $\Gamma$ on $G_{6}^{\text {irred }}$.

The mapping $\llbracket G_{2}^{* 3} * \cdot \rrbracket$ maps a function of $n-1$ independent momenta to a function of $n-3$ momenta, and consequently is not invertible. This is the reason why one cannot solve the ascending problem. As the input to the Eq. (6.2) is not of the form of power series in $g$, it is meaningless to demand that higher manypoint functions be expandable in powers of $g$.

\section{Concluding Remarks}

As has been seen in Chapters II and III, if $G_{4}$ is bounded as (1.4) and $g$ is sufficiently small, Newton-Kantorovich type successive approximations converge, and even the first approximation generates all the expected branch points, though their numerical coefficients are altered by higher approximations. There cannot be a continuum of solutions to the descending problem because of the Theorem 2 . Global uniqueness of solution, however, cannot be concluded from currently available techniques.

One cannot descend from arbitrary $G_{N}(N \geqq 6)$ even if the $G_{N}$ is suitably bounded (Chapter V). As to the uniqueness of $G_{n}(n<N)$ corresponding to a given $G_{N}$ that has at least one set of $G_{n}$ 's $(n N)$ satisfying relevant conditions, one can say that two sets of $G_{n}$ 's cannot be too close each other if a second set exists at all.

The method developed in the present article cannot be applied to the problem of dynamically generated mass (Maris, Herscovitz, and Jacob [17], Pagels [18]), because in our method one has to begin with renormalised mass and regularise the operator equation as in the Eq. (1.2). 
Now, one of the most important question of our approach to the quantum field theory is whether one can take $G_{4}$ as an input to a system of equations for many-point functions, while the Lagrangian specifies the form of those equations. As the input $G_{4}$ cannot be given in the form of a power series in $g$, it is meaningless to assume that higher many point functions can be expanded in powers of $g$ and consequently such an assumption cannot be used as criterion in choice of the solution of the ascending problem. One of the conceivable criteria for choice of the solution of the ascending problem is that $\left\|G_{n}^{\text {irred }}\right\| \rightarrow 0$ as $n \rightarrow \infty$. But we do not know whether there are inequivalent norms and whether the above criterion is sufficient for singling out the physically meaningful solution.

It should be noticed that in spite of non-linearity of the problem, we have not come to any condition for bifurcation of solutions. (The Krasnosel'skii school's theory [19] is not applicable to our complex Banach space.) On the other hand, it should be remembered that our problem is formulated in terms of complex valued functions of real variable, not in terms of complex analytic functions. This is an important difference from dispersion theories.

\section{References}

1. Taylor, J.G.: Lectures in Theoretical Physics, X, pp. 221-268. Boulder, Colorado, 1968

2. Landau,L.D., Abrikosov, A.A., Khalatnikov, I.M.: DAN SSSR 94, 497--500, 773-776, $1197-1200(1954)$

3. Elworthy, K.D., Tromba, A. J.: Proc. Symp. Pure Math. Amer. Math. Soc. 15, 45-94 (1969)

4. Earle, C.J., Hamilton, R.S.: Proc. of Symp. Pure Math. Amer. Math. Soc. 16, 61-65 (1970)

5. Petryshyn, W. V.: Proc. Symp. Pure Math. Amer. Math. Soc. 18, Part 1, 206-233 (1970)

6. Schiop, A.I.: Metode aproximative în analiza neliniară. Bucureşti: Editura Academiei RSR 1972

7. Jankó,B.: Rezolvarea ecuaţiilor operaţionale neliniare în spaţii Banach. Bucureşti: Editura Academiei RSR 1968

8. Vainberg, M.M.: Variational Methods for the Study of Non-linear Operators. San Francisco: Holden-Day 1964

9. Altman, M.: Contractor with Non-linear Majorant Functions and Equations in Banach Space, Louisiana State University Preprint (1973)

10. Collatz, L.: Arch. Rational Mech. Anal. 2, 66-75 (1958)

11. Mirakov, V.E.: Tr. Mosk. Fiz. Tekhn. Inst. 1, 204-213 (1958)

12. Jankó, B.: Stud. şi Cerc. Mat. 12, 301-308 (1961)

13. Jankó, B.: Stud. şi Cerc. Mat. 14, 265-271 (1963)

14. Jankó, B., Balazs, M.: Stud. şi Cerc. Mat. 18, 817-828 (1966)

15. Balazs, M., Jankó, B.: Stud. şi Cerc. Mat 20, 809-817 (1968)

16. Taylor,J.G., Yoshimura,T.: Which Green's Functions Equations? King's College (London) Preprint (1973)

17. Maris, Th. D., Herscovitz, V.E., Jacob, G.: Phys. Rev. Letters 12, 313-315 (1964)

18. Pagels, H.: Phys. Rev. D7, 2689-2698 (1973)

19. Krasnosel'skiǐ,M.A., Vainikko,G.M., Zabrě̌ko,P.P., Rutitskiǐ,Ya.B., Stetsenko,V.Ya.: Priblizhennoe reshenie operatornykh uravnenii, Moskva Izd. "Nauka" (1969), and earlier works cited therein 